\title{
Nuclear curvature energy in relativistic models
}

\author{
M. Centelles and X. Viñas \\ Departament d'Estructura i Constituents de la Matèria, Facultat de Física, Universitat de Barcelona, \\ Diagonal 647, E-08028 Barcelona, Spain
}

P. Schuck

Institut National de Physique Nucléaire et de Physique des Particules, Centre National de la Recherche Scientifique (CNRS-IN2P3), Université Joseph Fourier, Institut des Sciences Nucléaires, 53 Avenue des Martyrs, F-38026 Grenoble Cedex, France

(Received 9 May 1995)

\begin{abstract}
The difficulties arising in the calculation of the nuclear curvature energy are analyzed in detail, especially with reference to relativistic models. It is underlined that the implicit dependence on curvature of the quantal wave functions is directly accessible only in a semiclassical framework. It is shown that also in the relativistic models quantal and semiclassical calculations of the curvature energy are in good agreement.
\end{abstract}

PACS number(s): 21.10.Dr, 21.60. $-\mathrm{n}, 21.65 .+\mathrm{f}$

While there exists general agreement on the magnitude of the volume $\left(a_{v}\right)$ and surface $\left(a_{s}\right)$ energy coefficients in the nuclear mass formula

$$
E=a_{v} A+a_{s} A^{2 / 3}+\left(a_{c}-\frac{2 a_{s}^{2}}{K_{\infty}}\right) A^{1 / 3}+\cdots
$$

( $K_{\infty}$ is the bulk incompressibility), the curvature energy contribution $a_{c}$ has been up to now a controversial subject. On the one hand, all the past theoretical calculations (most of them based on semiclassical methods) have reported a value for the $a_{c}$ coefficient around $10 \mathrm{MeV}$, whereas the empirical value extracted from fits to nuclear binding energies and fission barriers is compatible with zero. Very recently, Myers and Swiatecki [1] have provided an explanation to this socalled curvature energy puzzle and conclude that the theoretical estimates of $a_{c}$ are probably quite realistic.

On the other hand, a recent paper by Von-Eiff et al. [2] dealing with the analysis of nuclear surface properties in the relativistic mean field theory (RMFT) presents at the end, for the first time in the literature, a few relativistic quantal calculations of the curvature energy. The authors of [2] compare their results with the corresponding semiclassical calculations of Ref. [3], finding a large discrepancy which is interpreted as an indication that semiclassical approaches to the curvature energy might not be sufficient within the relativistic framework [2]. Indeed $a_{c}$ is a very subtle quantity. The aim of the present paper is twofold: to give a clarifying outline, tailored to the relativistic model case, of the calculation of the curvature energy coefficient, and to show that in Ref. [2] there was a misinterpretation when comparing the relativistic quantal and semiclassical curvature energy results, which will be shown to be both in good agreement.

We start by shortly recalling some basic considerations (see also Refs. [3-7]). In the droplet model [8] the energy of an uncharged spherical nucleus with mass number $A$ is split into a volume and a surface part:

$$
E=a_{v} A+4 \pi \int_{0}^{\infty} d r r^{2}\left[\mathscr{E}(r)-a_{v} \rho(r)\right]
$$

where $a_{v}$ is the energy per particle in the bulk, $\mathscr{E}(r)$ and $\rho(r)$ stand for the local energy and particle densities, respectively, and the quantity $\mathscr{E}_{s}=\mathscr{E}-a_{v} \rho$ is called the surface energy density. Following Ref. [8], one writes Eq. (2) in terms of a variable $u=r-R$ which measures the distance from the equivalent sharp surface of radius $R$, expands (2) in powers of the curvature $\kappa(2 / R$ for spherical systems), and takes the limit $R \rightarrow \infty$, in which case the geometry becomes that of a semi-infinite system. The surface energy coefficient is given by the lowest order of the $\kappa$ expansion (corresponding to the plane surface):

$$
a_{s}=\left.4 \pi r_{\infty}^{2} \int_{-\infty}^{\infty} d z \mathscr{E}_{s}(z, \kappa)\right|_{\kappa=0},
$$

where the variable $u$ has been replaced with $z$, the coordinate perpendicular to the surface, and $r_{\infty}=\left(3 / 4 \pi \rho_{\infty}\right)^{1 / 3}$ is the nuclear matter radius (with $\rho_{\infty}$ the saturation density). The linear term in $\kappa$ gives the curvature energy coefficient

$$
\begin{aligned}
a_{c}=a_{c}^{\mathrm{geo}}+a_{c}^{\mathrm{dyn}}= & \left.8 \pi r_{\infty} \int_{-\infty}^{\infty} d z\left(z-z_{0}\right) \mathscr{E}_{s}(z, \kappa)\right|_{\kappa=0} \\
& +\left.8 \pi r_{\infty} \int_{-\infty}^{\infty} d z \frac{\partial \mathscr{E}_{s}(z, \kappa)}{\partial \kappa}\right|_{\kappa=0} .
\end{aligned}
$$

The constant $z_{0}=\left[\int_{-\infty}^{\infty} d z z \rho^{\prime}(z)\right] /\left[\int_{-\infty}^{\infty} d z \rho^{\prime}(z)\right]$ is the location of the effective sharp surface.

The two contributions to the curvature energy in (4) are called geometrical $\left(a_{c}^{\text {geo }}\right)$ and dynamical $\left(a_{c}^{\text {dyn }}\right)$. The geometrical contribution only involves the variation of $\mathscr{E}_{s}$ across the surface parallel to the $z$ axis. The dynamical part comes from the change of $\mathscr{E}_{s}$ by curvature when the plane surface is infinitesimally bent. Some confusion may arise since the numerical values of $a_{c}^{\text {geo }}$ and $a_{c}^{\text {dyn }}$ are not uniquely defined (only their sum is). This stems from the fact that the form of $\mathscr{E}(r)$ in (2) is not unique under the integral sign. Actually, $\mathscr{E}(r)$ can contain Laplace operators both explicitly and im- 
plicitly. One notorious example for the explicit dependence is the kinetic energy part of $\mathscr{E}(r)$ in the nonrelativistic case (units $\hbar=c=1$ are used):

$$
-\frac{1}{2 m} \sum_{\alpha} \int d \mathbf{r} \varphi_{\alpha}^{*}(\mathbf{r}) \Delta \varphi_{\alpha}(\mathbf{r})=\frac{1}{2 m} \sum_{\alpha} \int d \mathbf{r}\left|\boldsymbol{\nabla} \varphi_{\alpha}(\mathbf{r})\right|^{2} .
$$

We thus see that the explicit appearance of Laplace operators can be modified arbitrarily by partial integration. These different forms of $\mathscr{E}(r)$ leave, of course, the total curvature energy (as well as the surface energy) unchanged but the individual values for $a_{c}^{\text {geo }}$ and $a_{c}^{\text {dyn }}$ are modified, since the Laplace operator in the limit $R \rightarrow \infty$ reads as $d^{2} / d z^{2}$ $+\kappa d / d z$. In order not to obscure the discussion unnecessarily, we will now make one definite choice for the form of $\mathscr{E}(r)$ for which one may also find a certain logic: The fully quantal expression of $\mathscr{E}(r)$ should be such that there appear no explicit Laplace operators; i.e., all $\Delta$ 's have been eliminated by partial integrations. The dynamical contribution $a_{c}^{\mathrm{dyn}}$ to the curvature energy then entirely comes from the implicit curvature dependence of the wave functions from which $\mathscr{E}(r)$ is constructed. Indeed the Schrödinger equation contains the Laplacian, and thus the wave functions and the quantum-mechanical density matrix contain the curvature $\kappa$ in a nonexplicit way. Though in the relativistic problem the Dirac equation is linear in the $\boldsymbol{\nabla}$ operator, the elimination of the lower components in favor of the upper components also generates an implicit dependence of the relativistic wave functions on the Laplacian.

It is fortunate that semiclassical expansions in powers of $\hbar$, like the Wigner-Kirkwood and density functional (extended Thomas-Fermi) approaches, make such implicit $\kappa$ dependence of the quantal $\mathscr{E}(r)$ an explicit one via the Laplace operators which appear in the semiclassical series for $\mathscr{E}(r)$ [7]. At this step one again has to be very careful in order not to get mixed up. Actually, the Laplacians in the $\hbar$ expansion can also be eliminated in various ways by partial integrations, leading to a change in $a_{c}^{\text {geo }}$ and $a_{c}^{\text {dyn }}$ separately. If we are interested in a direct comparison with our above definition of $a_{c}^{\mathrm{dyn}}$ in the quantal case, it is clear that the Laplacians appearing in the $\hbar$ expansion should not be eliminated, since they reflect exclusively the implicit $\kappa$ dependence of the wave functions. As will be shown below, another important feature of a self-consistent calculation in the density functional theory is that the contribution to $a_{c}$ of the implicit $\kappa$ dependence of the density $\rho$ (and of the meson fields in the relativistic model), which stems from solving the EulerLagrange equations, exactly vanishes. We shall now exemplify all this with the relativistic model.

First, we address the calculation of $a_{c}^{\text {dyn }}$ in the quantal case. The starting point is the well-known Lagrangian of the RMFT including nonlinear couplings of the scalar field [9]. Using standard notation, in the relativistic Hartree approximation the local energy density $\mathscr{E}^{H}(\mathbf{r})$ of a neutral spherical nucleus reads

$$
\mathscr{E}^{H}=\sum_{\alpha} \varphi_{\alpha}^{\dagger}\left[-i \boldsymbol{\alpha} \cdot \boldsymbol{\nabla}+\beta m^{*}+g_{v} V_{0}-m\right] \varphi_{\alpha}+\mathscr{E}_{f},
$$

where $m^{*}=m-g_{s} \phi_{0}$ is the nucleon effective mass and

$$
\begin{aligned}
\mathscr{E}_{f}= & \frac{1}{2}\left[\left(\boldsymbol{\nabla} \phi_{0}\right)^{2}+m_{s}^{2} \phi_{0}^{2}\right]-\frac{1}{2}\left[\left(\boldsymbol{\nabla} V_{0}\right)^{2}+m_{v}^{2} V_{0}^{2}\right]+\frac{1}{3} b \phi_{0}^{3} \\
& +\frac{1}{4} c \phi_{0}^{4} .
\end{aligned}
$$

The single-particle wave functions $\varphi_{\alpha}$ and the scalar $\left(\phi_{0}\right)$ and vector $\left(V_{0}\right)$ meson fields are obtained by solving a set of coupled variational equations, namely, a Dirac equation for the nucleons,

$$
\frac{\delta \mathscr{E}^{H}}{\delta \varphi_{\alpha}^{\dagger}}=\left[-i \boldsymbol{\alpha} \cdot \boldsymbol{\nabla}+\beta m^{*}+g_{v} V_{0}-m\right] \varphi_{\alpha}=\varepsilon_{\alpha} \varphi_{\alpha},
$$

and standard Klein-Gordon equations for the mesons [9].

We remark that the quantal energy density $\mathscr{E}^{H}$ of Eq. (6) does not contain any explicit Laplace operators, so that $a_{c}^{\text {dyn }}$ comes only from the implicit curvature dependence of the wave functions and the meson fields. Then, from Eq. (4) one finds

$$
\begin{aligned}
a_{c, H}^{\mathrm{dyn}}= & 8 \pi r_{\infty} \int_{-\infty}^{\infty} d z\left[\sum_{\alpha}\left(\frac{\delta \mathscr{E}_{s}^{H}}{\delta \varphi_{\alpha}} \frac{d \varphi_{\alpha}}{d \kappa}+\frac{\delta \mathscr{E}_{s}^{H}}{\delta \varphi_{\alpha}^{\dagger}} \frac{d \varphi_{\alpha}^{\dagger}}{d \kappa}\right)\right. \\
& \left.+\frac{\delta \mathscr{E}_{s}^{H}}{\delta \phi_{0}} \frac{d \phi_{0}}{d \kappa}+\frac{\delta \mathscr{E}_{s}^{H}}{\delta V_{0}} \frac{d V_{0}}{d \kappa}\right]\left.\right|_{\kappa=0},
\end{aligned}
$$

with $\mathscr{E}_{s}^{H}=\mathscr{C}^{H}-a_{v} \rho$. Using $\rho=\Sigma_{\alpha} \varphi_{\alpha}^{\dagger} \varphi_{\alpha}$ and the variational equations for the meson fields $\delta \mathscr{E}^{H} / \delta \phi_{0}=\delta \mathscr{E}^{H} / \delta V_{0}=0$,

$$
\begin{aligned}
a_{c, H}^{\mathrm{dyn}}= & 8 \pi r_{\infty} \sum_{\alpha} \int_{-\infty}^{\infty} d z\left[\left(\frac{\delta \mathscr{E}^{H}}{\delta \varphi_{\alpha}}-a_{v} \varphi_{\alpha}^{\dagger}\right) \frac{d \varphi_{\alpha}}{d \kappa}\right. \\
& \left.+\left(\frac{\delta \mathscr{E}^{H}}{\delta \varphi_{\alpha}^{\dagger}}-a_{v} \varphi_{\alpha}\right) \frac{d \varphi_{\alpha}^{\dagger}}{d \kappa}\right]\left.\right|_{\kappa=0} .
\end{aligned}
$$

The equations $\delta \mathscr{E}^{H} / \delta \varphi_{\alpha}=\varepsilon_{\alpha} \varphi_{\alpha}^{\dagger}$ and $\delta \mathscr{E}^{H} / \delta \varphi_{\alpha}^{\dagger}=\varepsilon_{\alpha} \varphi_{\alpha}$ allow one to write

$$
a_{c, H}^{\mathrm{dyn}}=\left.8 \pi r_{\infty} \sum_{\alpha} \int_{-\infty}^{\infty} d z\left(\varepsilon_{\alpha}-a_{v}\right) \frac{d}{d \kappa}\left(\varphi_{\alpha}^{\dagger} \varphi_{\alpha}\right)\right|_{\kappa=0} .
$$

After $\kappa$ has been set equal to zero, the index $\alpha$ refers to the quantum numbers of the uncurved semi-infinite system, that is, $\alpha=\left(k_{z}, k_{\perp}, \eta\right)[10]$ with $k^{2}=k_{z}^{2}+k_{\perp}^{2}\left(0 \leqslant k \leqslant k_{F, \infty}\right)$ and $\eta= \pm 1$ the spin orientation. The Hugenholtz-Van Hove theorem tells us that for nuclear matter at equilibrium $a_{v}=\left(k_{F, \infty}^{2}+m_{\infty}^{* 2}\right)^{1 / 2}+g_{v} V_{0, \infty}-m$, where $k_{F}$ is the Fermi momentum and all quantities are evaluated at saturation. Finally, taking into account that the energy eigenvalues in semi-infinite nuclear matter are identical with the singleparticle energies in infinite nuclear matter [10], $\varepsilon_{\alpha}=\left(k^{2}+m_{\infty}^{* 2}\right)^{1 / 2}+g_{v} V_{0, \infty}-m$, one obtains

$$
\begin{aligned}
a_{c, H}^{\mathrm{dyn}}= & 8 \pi r_{\infty} \sum_{\alpha}\left[\sqrt{k^{2}+m_{\infty}^{* 2}}-\sqrt{k_{F, \infty}^{2}+m_{\infty}^{* 2}}\right] \\
& \times\left.\int_{-\infty}^{\infty} d z \frac{d}{d \kappa}\left(\varphi_{\alpha}^{\dagger} \varphi_{\alpha}\right)\right|_{\kappa=0} .
\end{aligned}
$$


In the nonrelativistic framework one would find a similar expression for the quantal $a_{c}^{\text {dyn }}$, with nonrelativistic singleparticle energies and wave functions. It is clear from Eq. (12) that in general the dynamical contribution to the curvature energy in a quantum-mechanical calculation is a nonvanishing quantity. Furthermore, it cannot be directly calculated since the dependence of the wave functions on $\kappa$ is unknown; i.e., we cannot evaluate the derivative $d\left(\varphi_{\alpha}^{\dagger} \varphi_{\alpha}\right) /\left.d \kappa\right|_{\kappa=0}$ in (12). As a consequence, the total curvature energy is not fully accessible within the quantal framework, and it is only its geometrical part $a_{c}^{\text {geo }}$ which can be directly calculated.

As mentioned above, when one resorts to a semiclassical formalism the implicit curvature dependence of the wave functions turns into an explicit one in the form of Laplace operators in the $\hbar$ series, so that one can certainly get a value for $a_{c}^{\text {dyn }}$ in the semiclassical context. We will illustrate this in our problem by considering the relativistic extended Thomas-Fermi (RETF) method [11], which is an extension of the relativistic Thomas-Fermi (RTF) approximation [9]. It incorporates gradient corrections of second order in $\hbar$ and thus it is more appropriate than the RTF approximation for the description of nuclear surface properties [3,12]. For an uncharged spherical nucleus, the RETF energy density $\varepsilon^{\operatorname{RETF}}(\mathbf{r})$ of the RMFT is

$$
\mathscr{E}^{\mathrm{RETF}}=\mathscr{E}_{0}+\mathscr{E}_{2}+g_{v} V_{0} \rho-m \rho+\mathscr{E}_{f}
$$

$\mathscr{E}_{f}$ has been defined in Eq. (7); $\mathscr{E}_{0}$ is the well-known RTF functional,

$$
\mathscr{E}_{0}=\frac{1}{4 \pi^{2}}\left[k_{F} \varepsilon_{F}^{3}+k_{F}^{3} \varepsilon_{F}-m^{* 4} \ln \frac{k_{F}+\varepsilon_{F}}{m^{*}}\right],
$$

and

$$
\begin{aligned}
\mathscr{E}_{2}= & C_{1}(\nabla \rho)^{2}+C_{2} \boldsymbol{\nabla} \rho \cdot \nabla m^{*}+C_{3}\left(\nabla m^{*}\right)^{2}+C_{4} \Delta \rho \\
& +C_{5} \Delta m^{*}
\end{aligned}
$$

is the correction of order $\hbar^{2}$. As usual the local Fermi momentum is related to the density via $k_{F}=\left(3 \pi^{2} \rho / 2\right)^{1 / 3}$, $\varepsilon_{F}=\sqrt{k_{F}^{2}+m^{* 2}}$, and the functions $C_{i}$ are defined as follows:

$$
\begin{gathered}
C_{1}\left(k_{F}, m^{*}\right)=\frac{\pi^{2}}{48 k_{F}^{3} \varepsilon_{F}^{2}}\left[2 k_{F}\left(1+2 \frac{\varepsilon_{F}^{2}}{k_{F}^{2}}\right) \ln \frac{k_{F}+\varepsilon_{F}}{m^{*}}\right. \\
\left.-\varepsilon_{F}\left(3+2 \frac{k_{F}^{2}}{\varepsilon_{F}^{2}}\right)\right], \\
C_{2}\left(k_{F}, m^{*}\right)=\frac{1}{6 \varepsilon_{F}^{2}}\left[\frac{k_{F}^{2}}{m^{*} \varepsilon_{F}}+\frac{m^{*}}{k_{F}} \ln \frac{k_{F}+\varepsilon_{F}}{m^{*}}\right], \\
C_{3}\left(k_{F}, m^{*}\right)=\frac{k_{F}^{3}}{12 \pi^{2} \varepsilon_{F}^{3}}\left(2+3 \frac{\varepsilon_{F}^{2}}{k_{F}^{2}}\right)\left[1-\frac{\varepsilon_{F}}{k_{F}} \ln \frac{k_{F}+\varepsilon_{F}}{m^{*}}\right], \\
C_{4}\left(k_{F}, m^{*}\right)=\frac{1}{12 \varepsilon_{F}}\left[1-2 \frac{\varepsilon_{F}}{k_{F}} \ln \frac{k_{F}+\varepsilon_{F}}{m^{*}}\right] \\
C_{5}\left(k_{F}, m^{*}\right)=\frac{m^{*}}{6 \pi^{2}}\left[\frac{k_{F}}{\varepsilon_{F}}-\ln \frac{k_{F}+\varepsilon_{F}}{m^{*}}\right]
\end{gathered}
$$

Equations (13)-(20) are the semiclassical counterpart (to order $\hbar^{2}$ ) of the relativistic quantal energy density (6). We insist that no partial integrations of gradients or Laplacians have been performed in the expression given above for $\mathscr{E}_{2}$. Therefore, the explicit Laplace operators in Eq. (15) display exclusively the implicit dependence of the quantal wave functions on the curvature $\kappa$, which was hidden in $\mathscr{E}^{H}$. Note in passing that this is the first time that the functional $\mathscr{E}_{2}$ of the RETF method is given as in Eqs. (15)-(20), since in the previous literature it had always been published in the form which results after eliminating $\Delta \rho$ and $\Delta m^{*}$ by partial integrations $[3,11,12]$. The RETF variational density and meson fields are obtained by solving the Euler-Lagrange equation

$$
\frac{\delta \mathscr{E}^{\mathrm{RETF}}}{\delta \rho}=\varepsilon_{F}-m+g_{v} V_{0}+\frac{\delta \mathscr{E}_{2}}{\delta \rho}=\lambda,
$$

with $\lambda$ the chemical potential, and Klein-Gordon equations which have the same form as in the quantal case (see Ref. [3] for details).

Applying Eq. (4) to the surface energy density $\mathcal{E}_{s}^{\mathrm{RETF}}=\mathcal{E}^{\mathrm{RETF}}-a_{v} \rho$, we get the semiclassical value for the dynamical part of the curvature energy:

$$
\begin{aligned}
a_{c, \mathrm{RETF}}^{\mathrm{dyn}}= & 8 \pi r_{\infty} \int_{-\infty}^{\infty} d z\left[C_{4}\left(k_{F}, m^{*}\right) \frac{d \rho}{d z}\right. \\
& \left.+C_{5}\left(k_{F}, m^{*}\right) \frac{d m^{*}}{d z}\right]\left.\right|_{\kappa=0} \\
& +8 \pi r_{\infty} \int_{-\infty}^{\infty} d z\left[\left(\frac{\delta \mathscr{E}^{\mathrm{RETF}}}{\delta \rho}-a_{v}\right) \frac{d \rho}{d \kappa}\right. \\
& \left.+\frac{\delta \mathscr{E}^{\mathrm{RETF}}}{\delta \phi_{0}} \frac{d \phi_{0}}{d \kappa}+\frac{\delta \mathscr{E R E T F}^{\mathrm{RET}}}{\delta V_{0}} \frac{d V_{0}}{d \kappa}\right]\left.\right|_{\kappa=0} .
\end{aligned}
$$

The first two lines of (22) originate from the $\Delta$ operators present in $\mathscr{E}_{2}$ [we have used that $\partial(\Delta A) /\left.\partial \kappa\right|_{\kappa=0}$ $=d A /\left.d z\right|_{\kappa=0}$, with $\left.A=\rho, m^{*}\right]$. The last two lines represent the contribution of the implicit $\kappa$ dependence: The solutions $\rho, \phi_{0}$, and $V_{0}$ of (21) and of the Klein-Gordon equations depend in an implicit way on curvature (because those equations contain Laplacians). However, as mentioned earlier, this implicit $\kappa$ dependence does not play any role in the semiclassical curvature energy. First, for the self-consistent density and fields the chemical potential $\lambda$ equals the energy per particle $a_{v}$ of saturating nuclear matter. Second, the energy density is stationary with respect to variations of the density and the fields:

$$
\frac{\delta \mathscr{E}^{\mathrm{RETF}}}{\delta \rho}-\lambda=\frac{\delta \mathscr{E}^{\mathrm{RETF}}}{\delta \phi_{0}}=\frac{\delta \mathscr{E}^{\mathrm{RETF}}}{\delta V_{0}}=0 .
$$

Consequently, the integrand of the last two lines of (22) vanishes and $a_{c \text {, RETF }}^{\text {dy }}$ is simply given by the first two lines of this equation. Since we have made sure not to perform in $\mathscr{E}_{2}$ any rearrangement of $\Delta$ operators by partial integrations, it now is clear that Eq. (22) is the quantity which corresponds directly to the quantal $a_{c, H}^{\text {dyn }}$ of Eq. (12). Similarly, it will be valid to compare $a_{c \text {, RETF }}^{\text {geo }}$ with $a_{c, H}^{\mathrm{geo}}$ arising from the quantal functional (6) only if we employ Eq. (15) for $\mathscr{E}_{2}$. It should 
TABLE I. Contributions to the curvature energy calculated in the relativistic quantal Hartree approach and in the semiclassical RETF and RTF approximations (see text), for the parameter sets of Ref. [2] which have been labeled by their incompressibility $K_{\infty}$ (the remaining saturation properties are $a_{v}=-15.75 \mathrm{MeV}, \rho_{\infty}=0.16$ $\mathrm{fm}^{-3}$, and $m_{\infty}^{*} / m=0.55$, with $m_{s}=400 \mathrm{MeV}$ ). All quantities are in $\mathrm{MeV}$.

\begin{tabular}{lccccc}
\hline \hline$K_{\infty}$ & $a_{c, H}^{\text {geo }}[2]$ & $a_{c, \text { RETF }}^{\text {geo }}$ & $a_{c, \text { RETF }}^{\text {dyn }}$ & $a_{c, \text { RETF }}$ & $a_{c, \text { RTF }}[2]$ \\
\hline 200 & 13.87 & 14.19 & 14.84 & 29.03 & 28.56 \\
250 & 12.70 & 12.79 & 14.84 & 27.63 & 27.57 \\
300 & 12.15 & 11.71 & 14.84 & 26.54 & 26.98 \\
\hline \hline
\end{tabular}

be noted that in the simpler RTF approach where the $\hbar^{2}$ correction $\mathscr{E}_{2}$ and therefore the corresponding $\Delta$ operators are neglected we have $a_{c, \mathrm{RTF}}^{\mathrm{dyn}}=0$, and thus $a_{c, \mathrm{RTF}}=a_{c, \mathrm{RTF}}^{\text {geo }}$.

In Table I we present the values of $a_{c}^{\text {geo }}$ in the quantal and RETF approaches for the same three parameter sets of the relativistic model that were considered in Table IV of Ref. [2] (from which we have extracted $a_{c, H}^{\mathrm{geo}}$ ). We see that there is close agreement between the results of both approaches, as in the nonrelativistic case [7]. Also given are the values for $a_{c, \text { RETF }}^{\text {dyn }}$ to which no quantal counterparts exist, and the total curvature energy $a_{c, \mathrm{RETF}}=a_{c, \mathrm{RETF}}^{\mathrm{geo}}+a_{c, \mathrm{RETF}}^{\mathrm{dyn}}$. It is worth mentioning that in the nonrelativistic framework the semiclassical $a_{c}^{\text {dyn }}$ is analytical [7] and only depends on nuclear matter properties (the saturation density and effective mass). We have performed numerical tests that indicate that this also happens in the relativistic model, but we have not been able to obtain an analytical result for Eq. (22). Though the RTF approximation misses the individual values in the separation of the curvature energy into dynamical and geometrical parts $\left(a_{c, \mathrm{RTF}}^{\mathrm{dyn}}=0\right)$, we have also included $a_{c, \mathrm{RTF}}$ in Table I to show that the RTF method provides a reasonable estimate for the total value, which is close to the RETF one. This is not surprising as we have checked that in the RETF calculation the contribution of order $\hbar^{2}$ to $a_{c \text {, RETF }}^{\text {geo }}$, due to the functional $\mathscr{E}_{2}$ of Eq. (15), almost cancels out the dynamical part $a_{c \text {, RETF }}^{\text {dyn }}$, so that the net correction of order $\hbar^{2}$ to the curvature energy is small as compared with the Thomas-Fermi contribution (order $\hbar^{0}$ ).
One should not attach a special significance to the fact that the magnitude of $a_{c, \text { RETF }}$ or $a_{c, \text { RTF }}$ in Table I is about a factor of 3 larger than the usual values of $\sim 10 \mathrm{MeV}$. This is due to the small scalar mass $m_{s}=400 \mathrm{MeV}$ of these parameter sets, which was chosen in Ref. [2] so as to minimize the influence of Friedel oscillations and spin-orbit effects in the quantal density distributions. Current parameter sets of the relativistic model have $m_{s}$ around $500 \mathrm{MeV}$ and yield better values for $a_{c}$; see Ref. [3].

We now explain where the comparison made in Ref. [2] of their quantal results for $a_{c, H}^{\text {geo }}$, obtained from the functional (6), with the RETF values for the curvature energy published in Ref. [3] went wrong. As discussed in [3] the RETF calculations were made with the energy density $\mathscr{E}_{2}^{*}$ which one obtains from Eq. (15) once the Laplacians have been removed by suitable partial integration (a starred quantity means that it is calculated as in [3] using the functional $\left.\mathscr{E}_{2}^{*}\right)$. In practice, if one only wants the total curvature energy, $\mathscr{E}_{2}^{*}$ has the advantage that $a_{c, \text { RETF }}^{\text {dyn* }}=0$ and $a_{c, \text { RETF }}^{\text {geo* }}$ $=a_{c, \mathrm{RETF}}^{*}=a_{c, \mathrm{RETF}}$; i.e., for $\mathscr{E}_{2}^{*}$ the total curvature energy coincides with the geometrical part. Unfortunately, when comparing the semiclassical and quantal results Von-Eiff et al. [2] took $a_{c, \mathrm{RETF}}^{\mathrm{geo} *}$ as the quantity which should correspond to $a_{c, H}^{\mathrm{geo}}$, and therefore found a striking disagreement between them. In doing this comparison it was overlooked that $a_{c, H}^{\text {geo }}$ leaves out the implicit $\kappa$ contribution from the wave functions, i.e. $a_{c, H}^{\mathrm{dyn}}$, whereas its semiclassical counterpart is included in $a_{c, \text { RETF }}^{\text {geo } *}$. Such disagreement disappears if the proper comparison is made: Indeed their values for $a_{c, H}^{\text {geo }}$ agree very nicely with our values for $a_{c, \text { RETF }}^{\text {geo }}$ listed in Table I.

In conclusion, we have tried to carefully point out the subtleties and pitfalls when dealing with the curvature energy, and to make clear the fact that the implicit curvature content of the quantal wave functions can be directly evaluated only in a semiclassical framework which includes, at least, second order corrections in $\hbar$. We have shown that also in the relativistic models there is full consistency between quantum-mechanical and semiclassical results.

This work was supported by the DGICYT (Spain) under Grant No. PB92-0761, the IN2P3-CICYT Collaboration, and Contract No. ERBCHRXCT920075 of the Human Capital and Mobility program.
[1] W. D. Myers and W. J. Swiatecki, Lawrence Berkeley Laboratory Report No. 36557, 1995, Nucl. Phys. A (submitted).

[2] D. Von-Eiff, W. Stocker, and M. K. Weigel, Phys. Rev. C 50, 1436 (1994).

[3] M. Centelles and X. Viñas, Nucl. Phys. A563, 173 (1993).

[4] M. Brack, C. Guet, and H.-B. Håkansson, Phys. Rep. 123, 275 (1985).

[5] J. Treiner and H. Krivine, Ann. Phys. (N.Y.) 170, 406 (1986).

[6] W. Stocker, J. Bartel, J. R. Nix, and A. J. Sierk, Nucl. Phys. A489, 252 (1988).
[7] M. Durand, P. Schuck, and X. Viñas, Z. Phys. A 346, 87 (1993).

[8] W. D. Myers and W. J. Swiatecki, Ann. Phys. (N.Y.) 55, 395 (1969).

[9] B. D. Serot and J. D. Walecka, Adv. Nucl. Phys. 16, 1 (1986); B. D. Serot, Rep. Prog. Phys. 55, 1855 (1992).

[10] D. Hofer and W. Stocker, Nucl. Phys. A492, 637 (1989).

[11] M. Centelles, X. Viñas, M. Barranco, and P. Schuck, Ann. Phys. (N.Y.) 221, 165 (1993).

[12] C. Speicher, E. Engel, and R. M. Dreizler, Nucl. Phys. A562, 569 (1993). 